\title{
Enhancing On-Demand Multicast Routing Protocols using Mobility Prediction in Mobile Ad-hoc Network
}

\author{
Nermin Makhlouf
}

\begin{abstract}
A Mobile Ad-hoc Network (MANET) is a selforganizing wireless communication network in which mobile devices are based on no infrastructure like base stations or access points. Minimal configuration and quick deployment make ad-hoc networks suitable for emergency situations like disaster recovery or military conflict.

Since node mobility may cause links to be broken frequently, a very important issue for routing in MANETs is how to set reliable paths which can last as long as possible. To solve this problem, non-random behaviors for the mobility patterns that mobile users exhibit are exploited.

This paper introduces a scheme to improve On-Demand Multicast Routing Protocol (ODMRP) performances by using mobility prediction.
\end{abstract}

Keywords - GPS, LET, MANET, mobility prediction, ondemand routing protocol.

\section{INTRODUCTION}

Since a mobile ad-hoc network consists of wireless hosts that may move often, the protocol must adapt to frequent changing network topologies. The increased mobility of adhoc nodes presents a challenging issue for protocol design. Various routing schemes have been proposed for ad-hoc networks [1], [2], [3], [4]. In order to improve routing protocol performance, there are two schemes that utilize location information (for instance, obtained using the global positioning system). The first scheme is Location Aided Routing (LAR) that uses location information obtained from the Global Positioning System (GPS) [5], [6] to limit the search for a new path to a smaller request region of the adhoc network. Another location-based routing protocol is Distance Routing Effect Algorithm for Mobility (DREAM) [7]. DREAM updates routing table periodically.

Multicasting is one of the most significant areas in the field of networking. With the growing technology and popularity of the internet, applications that need multicasting (e.g., video conferencing) are becoming more prevalent. Multicast plays a main role in ad-hoc networks because of the concept of teams and the need to show data to hold conferences between them [15].

In this paper enhancement of the On-Demand Multicast Routing Protocol using Mobility Prediction ODMRP-MP is presented. ODMRP uses on-demand routing techniques to

Manuscript received July 30, 2014. Revised August 21, 2014.

Nermin Makhlouf is with the Department of Telecommunications, Brno University of Technology, Czech Republic,

e-mail: xmakh100@stud.feec.vutbr.cz. overcome channel overhead and improve scalability. It uses the concept of forwarding group [8] to build a forwarding mesh for each multicast group. By using a mesh instead of a tree, the disadvantages of multicast trees in mobile wireless networks (e.g., intermittent connectivity, traffic concentration, frequent tree reconfiguration, non-shortest path in a shared tree, etc.) are avoided.

The paper is organized as follows. The second section shows the mechanism used for predicting the Link Expiration Time (LET). The third section presents method to enhance ondemand multicast routing protocol based on mobility prediction. The fourth section describes performance evaluation. The final section is conclusion.

\section{Mobility PREDICTION MeChanismS}

To improve various unicast and multicast ad-hoc routing protocols in [9] a prediction mechanism for link expiration time (LET) between two ad-hoc nodes has been studied. We assume a non-random movement pattern [10]. We also assume that all nodes in the network have their clock synchronized (e.g. by using the GPS clock itself). And two nodes $i$ and $j$ are within the transmission range of each other. We can calculate the period of time two neighboring nodes will remain connected by using mobility parameters of these two nodes (e.g. speed, direction, etc.). If the nodes $i$ and $j$ at locations $\left(x_{i}, y_{i}\right)$ and $\left(x_{j}, y_{j}\right)$ are moving at speed $v_{i}$ and $v_{j}$ respectively, and $\theta_{i}$ and $\theta_{j}$ are the moving directions of nodes $i$ and $j$, respectively, then the link expiration time is:

$$
D_{t}=\frac{-(\mathrm{ab}+\mathrm{cd})+\sqrt{\left(a^{2}+c^{2}\right) r^{2}-(a d-b c)^{2}}}{a^{2}+c^{2}}
$$

Where,

$$
\begin{aligned}
& a=v_{i} \cos \theta_{i}-v_{j} \cos \theta_{j} \\
& b=x_{i}-x_{j} \\
& c=v_{i} \sin \theta_{i}-v_{j} \sin \theta_{j} \\
& d=y_{i}-y_{j} .
\end{aligned}
$$

If the nodes $i$ and $j$ are traveling at the same speed and the same direction $\left(v_{i}=v_{j}, \theta_{i}=\theta_{j}\right)$, the link expiration time is unlimited. After predicting the LETs of all links of a route the minimum value of LETs is the Route Expiration Time (RET). This allows rebuilding route before route failure. The prediction of link expiration time may not be accurate because GPS may not work correctly in particular situations (e.g. indoor, fading, etc.) 


\section{MobiLity PREDICTION BASED MULTICAST RoUting PROTOCOLS}

\section{A. On Demand Multicast Routing Protocol with Mobility Prediction (ODMRP-MP)}

In ODMRP, the source creates and updates group membership and multicast routes on demand (nonperiodically) to reduce the channel overhead. ODMRP depends on the concept of forwarding group [8], [13].

The forwarding group is a set of nodes that is in charge of forwarding multicast data on the shortest paths between any member pairs. It has two cycles: a request cycle and a reply cycle. When a source wants to send packets, it periodically broadcasts the join request, which includes a member advertising packet and data, to the entire network to update the membership information and the routes. When a node receives a non-duplicate join request, it saves the source ID in its routing table and then it rebroadcasts the join request. When a desired multicast receiver receives the join request, it builds a join table which is broadcasted to neighboring nodes. When a node receives the join table it understands that it is on the route to the source in case that the next node ID of one of the entries has the same ID. This node is a part of the forwarding group, thus it builds its own join table which is also propagated to neighboring nods after setting the Forwarding Group Flag (FG Flag). Each part of the forwarding group propagates the join table until it reaches the multicast source via the shortest path. This method updates the routes from sources to receivers and builds the forwarding group. Fig. 1 shows the request and the reply cycles of ODMRP. After building the forwarding group and routes, a source can propagate packets to receivers by elected routes and forwarding. When a node receives the multicast data packet, it forwards the multicast data packet if the following two conditions are true: the multicast packet is not a duplicate, and the setting of the FG-Flag for the multicast group is still available. Nodes in the forwarding group will be non-forwarding nodes if they have not received join tables before their FG-Flag are expired. No control packets need to be sent to join or leave the group.

However, ODMRP discovers multicast routes only in the presence of data packets to be delivered to a multicast destination. Route discovery is based on request and reply cycles where multicast route information is stored in all intermediate nodes on the multicast path.

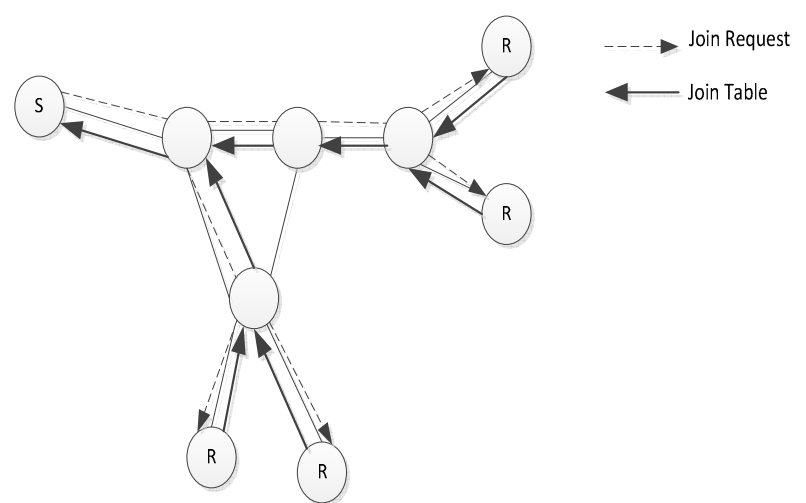

Fig. 1 The request and the reply cycles of ODMRP

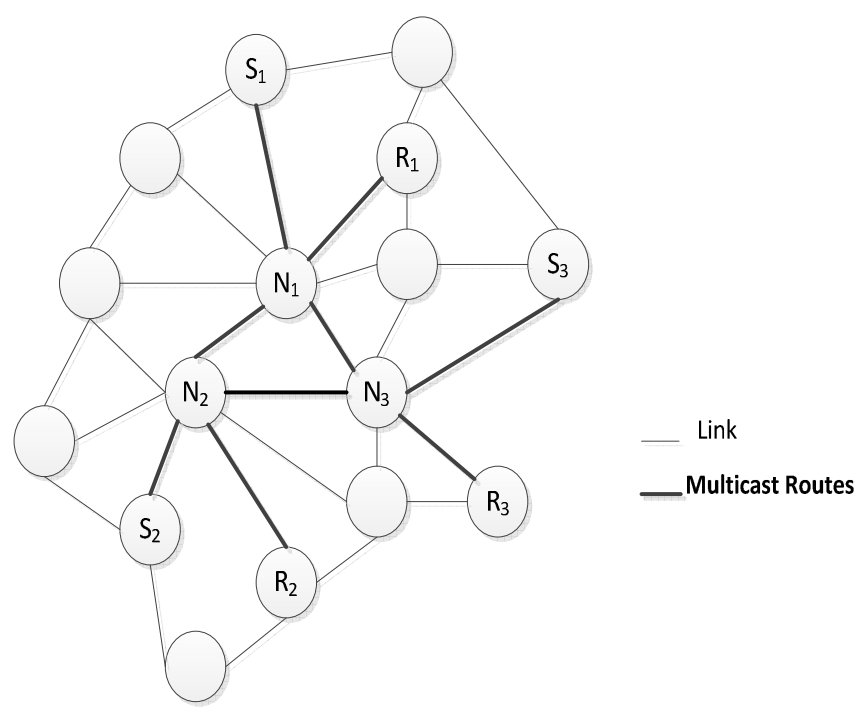

Fig. 2 An example of the ODMRP.

For example, there are three sources $\left(\mathrm{S}_{1}, \mathrm{~S}_{2}, \mathrm{~S}_{3}\right)$ as shown in Fig. 2 These sources send multicast data packets to three receivers $\left(R_{1}, R_{2}, R_{3}\right)$ through the forwarding group $\left(N_{1}, N_{2}\right.$, $N_{3}$ ). The route from $S_{1}$ to $R_{3}$ is $S_{1}, N_{1}, N_{3}, R_{3}$. If the route between $N_{1}$ and $N_{3}$ is broken, in ODMRP, the route $S_{1}, N_{1}$, $N_{2}, N_{3}, R_{3}$ will be in exchange for the route $S_{1}, N_{1}, N_{3}, R_{3}$. While in a tree configuration, the route from $S_{1}$ to $R_{3}$ is broken until the tree is reconfigured.

An example of join table forwarding is illustrated in Fig. 3 Multicast receivers R1 and R2 send their join tables to multicast sources S1 and S2 through N1. Multicast receiver $\mathrm{R}_{3}$ sends its join table to $\mathrm{S}_{1}$ through $\mathrm{N}_{1}$ and to $\mathrm{S}_{2}$ through $\mathrm{N}_{2}$. When source $S_{2}$ wants to send data to $R_{3}$, it broadcasts the join request. When node $\mathrm{N}_{2}$ receives the join request, it saves the source ID in its routing table and then it rebroadcasts the join request. A desired receiver $R_{3}$ receives the join request; it builds a join table which is broadcasted to the neighboring nodes. When an intermediate node $\mathrm{N}_{2}$ receives the join table of $R_{3}$, it sets the FG-Flag and builds its join table because the next node ID of one of the entries is the same of its ID.

Note that the channel overhead is decreased in the case where node N1 broadcasts the join table once even though it receives three join tables from three receivers R1, R2, R3

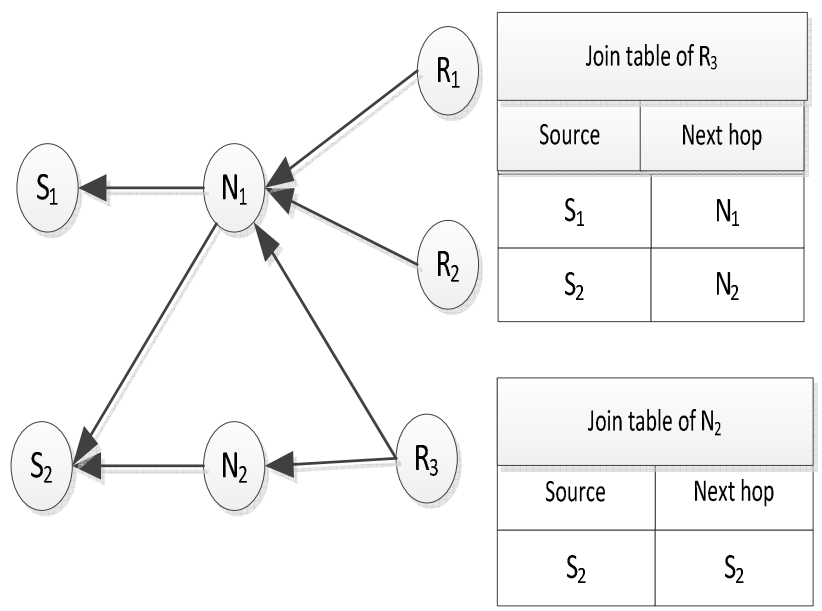

Fig. 3 An example of a join table forwarding. 
because these multicast receivers have the same links to the source. ODMRP requires periodic flooding of join request to build and refresh routes which often causes contention and collisions.

Thus it is important to find the optimal flooding interval. Here a scheme adapts the flooding interval to mobility and location information.

\section{B. Applying mobility prediction}

We predict the period of time two neighboring nodes will remain connected by using mobility parameters of these two nodes provided by GPS [11]. With the predicted time of route disconnection, join request is only flooded when route breaks of ongoing data sessions are imminent. A join request is tailed by the location, speed and direction of the source. The source sets the minimum LET field to the maximum LET value. The neighboring node, which receives a join request, predicts the link expiration time between itself and the previous hop using the equation (1).

It rebroadcasts the join request, but this join request will include the minimum between this value and the MIN LET indicated by the receiving join request, this minimum value is the Rout Expiration Time (RET). The node also updates the location and mobility information field according to its own information. If a forwarding group node receives multiple Join tables with different RET values from the same source to multiple receivers, it selects the minimum RET among them and sends its own join table with the chosen RET value attached. Then the source can build new routes by flooding a join request when route breaks of ongoing data sessions are imminent (i.e. before the minimum RET approaches). And receivers only send Join Tables after receiving join request.

The selection of the MIN REFRESH INTERVAL and the MAX REFRESH INTERVAL should be adaptive to network situations (e.g., traffic type, traffic load, mobility pattern, mobility speed, channel capacity, etc.)

In this scheme, instead of using the minimum delay path, we can choose the most stable route (i.e. the one with the largest RET). An example of route selection algorithms is presented in Fig. 4. Two routes are available from the source $S$ to the receiver $\mathrm{R}$. Route 1 has a path of (S-A-B-C-R) and route 2 has a path of (S-A-D-C-R). The route expiration time of route 1 is $2(\min .(3 ; 2 ; 4 ; 5)=2)$ while that of route 2 is 4 (min. $(3 ; 5 ; 1 ; 5)=1)$. The receiver selects the route with the maximum RET, and hence route 1 is selected.

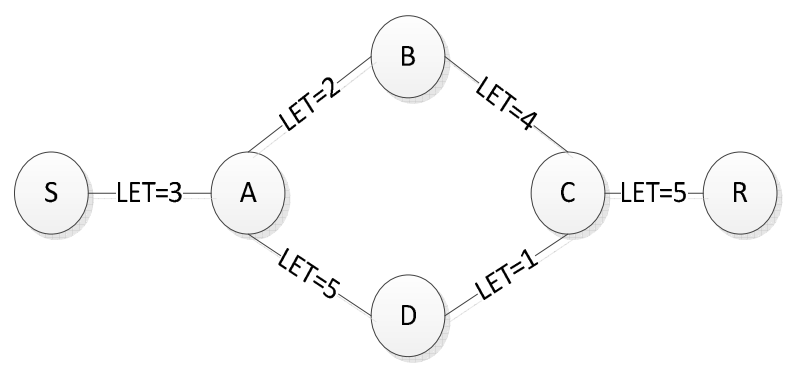

Fig. 4 Route selection example
The multicast receiver should waits for a convenient period of time to receive all possible routes and then the route which has the maximum RET is selected to be included in a join table.

\section{The prediction error}

The route expiration time prediction could become incorrect because a node can accelerate, decelerate and change its direction while it is traveling. Also the mobility information obtained from GPS may not always be accurate.

If we suppose that there is no sudden change of direction and the mobility information obtained from GPS is accurate, the predicted route expiration time of a node could be always accurate.

\section{PERFORMANCE EVALUATION}

The simulator has been implemented within the Global Mobile Simulation (GloMoSim) library [14]. The simulated environment consists of 50 wireless mobile nodes roaming in a 1000 meters x 1000 meters flat space for 600 seconds of simulated time. The radio transmission range is 250 meters and channel capacity was $2 \mathrm{Mbit} / \mathrm{sec}$. each packet has a payload of 512 bytes Mbits/sec.

Random waypoint model [12] is used as the mobility model. It means that a node randomly selects a destination and moves towards that destination at a predefined speed. In the MAC layer we use Carrier Sense Multiple Access/Collision Avoidance (CSMA/CA) scheme with acknowledgments.

In order to evaluate ODMRP, we use the following metrics:

Packet Delivery Ratio (PDR) as a function of speed: the ratio of data packets that are successfully delivered to a destination compared to the number of data packets that have been sent out by the sender when the speed varies from 0 to $20 \mathrm{~km} / \mathrm{h}$. Mathematically, PDR can be defined as:

$P D R=\frac{\sum \text { data packets received }}{\sum \text { data packets sent }}$

Number of Total packets transmitted per data packet delivered (NT): The number of all packets (i.e. join requests, join tables, data, and active acknowledgments) packets transmitted divided by the number of data packet delivered to destinations when the speed varies from 0 to $20 \mathrm{~km} / \mathrm{h}$. This measure shows the efficiency in terms of channel access. Mathematically, it can be defined as:

$N T=\frac{\sum \text { all packets transmitt ed }}{\sum \text { data packets delivered }}$

Fig. 5 shows the packet delivery ratio as a function of mobility speed. A comparison was made with ODMRP and ODMRP with Mobility Prediction (ODMRP-MP). 


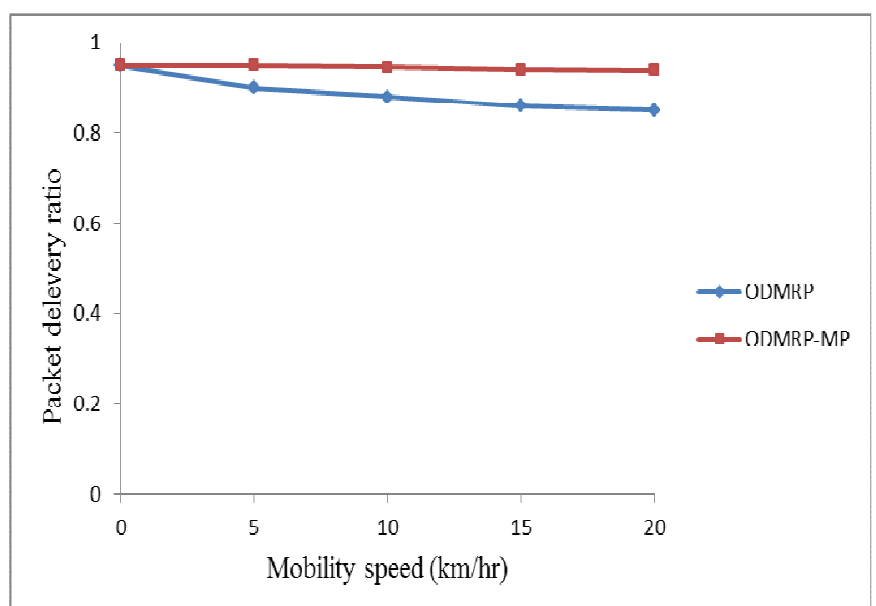

Fig. 5 Packet Delivery Ratio (PDR) as a function of speed

We inferred that ODMRP-MP is more effective compared to ODMRP because ODMRP-MP has a very high packet delivery ratio (over $90 \%$ ) regardless of speed. While in ODMRP the packet delivery degrades rapidly by the increase in the speed. This result is logical because ODMPRMP reconstructs the routes before topology changes, thus most data are received without being lost.

Fig. 6 shows the number of total packets transmitted per data packet delivered as a function of mobility speed for both ODMRP and ODMRP-MP. In ODMRP-MP, in order to adapt to the increasing speed, more control packets need to be sent. ODMRP-MP therefore delivers a high part of the data to destinations regardless of speed. Thus the number of total packets transmitted per data packet delivered increases with mobility speed. The number of total packets transmitted per data packet delivered for ODMRP almost does not change with speed.

\section{CONCLUSION}

In summary, we have presented the use of mobility prediction with ODMRP to overcome channel overhead and to improve scalability. ODMRP-MP predicts the time of route disconnection, thus it reconstructs the routs when route breaks of ongoing data sessions are imminent (i.e. before the minimum RET approaches).

It uses the concept of forwarding group [7] to build a forwarding mesh for each multicast group.

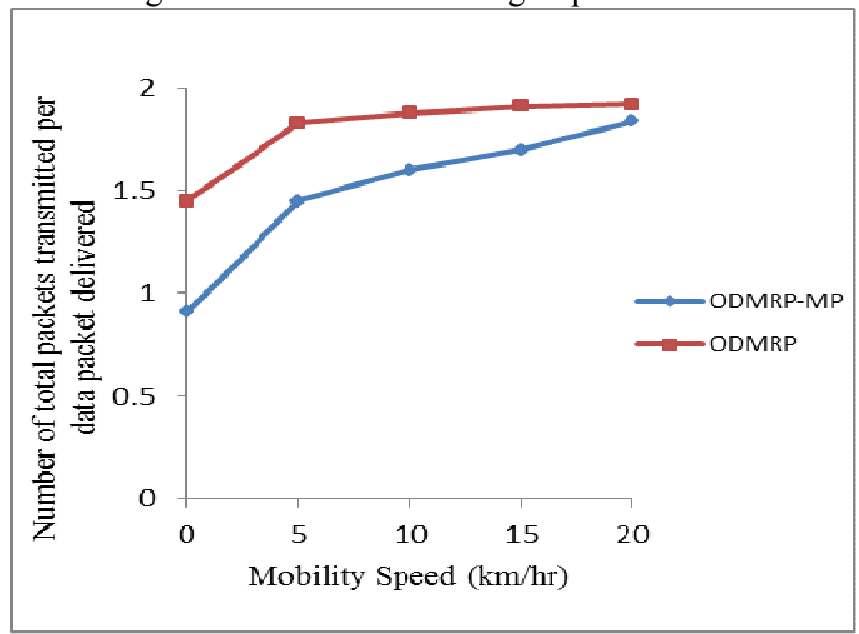

Fig. 6 Number of total packets transmitted per data packet delivered as a function of speed
Compared with trees the disadvantages of multicast trees in mobile wireless networks (e.g., intermittent connectivity, traffic concentration, frequent tree reconfiguration, nonshortest path in a shared tree, etc.) are avoided. According to simulation results with mobility prediction enhancements, more data packets were delivered to destinations. The control packets were used more efficiently.

\section{ACKNOWLEDGMENT}

This research is funded by project OPVK CZ.1.07/2.2.00/28.0062 "Joint activities of BUT and VSBTUO while creating the content of accredited technical courses in ICT".

\section{REFERENCES}

[1] M. S. Corson and A. Ephremides, "A distributed routing algorithm for mobile wireless networks," Wireless Networks, vol. 1, no. 1, pp. 61-81, 1995.

[2] S. Murthy and J. J. Garcia-Luna-Aceves, "An efficient routing protocol for wireless networks," Mobile Networks and Applications, vol. 1, no. 1, pp. 183-197, 1996.

[3] C.-K. Toh, "Associativity-Based Routing for Ad-hoc Mobile Networks," Wireless Personal Communications, vol. 4, no. 2, pp. 103-139, 1997.

[4] C. E. Perkins and E. M. Royer, "Ad-hoc On Demand Distance Vector Routing," in IEEE WMCSA 99, New Orleans, 1999.

[5] E. D. Kaplan and C. Hegarty, Understanding GPS: Principles and Applications, Second Edition, Boston: Artech House, 2005.

[6] S. Basagni, I. Chlamtac, V. R. Syrotiuk and B. A. Woodward, "A distance routing effect algorithm for mobility (DREAM)," in the 4th annual ACM/IEEE international conference on Mobile computing and networking, New York, 1998.

[7] M. S. Grewal, L. R. Weill and A. P. Andrews, Global positioning systems, inertial navigation, and integration, 2nd Edition, new gersey: Wiley, 2007.

[8] W. Su and M. Gerla, "IPv6 flow handoff in ad-hoc wireless networks using mobility prediction," in Global Telecommunications Conference, Rio de Janeireo, 1999.

[9] T. S. Rappaport, Wireless Communications: Principles and Practice, Prentice Hall, 1995.

[10] C.-C. Chiang, M. Gerla and L. Zhang, "Forwarding Group Multicast Protocol (FGMP) for Multihop, Mobile Wireless Networks," Cluster Computing, vol. 1, no. 2, pp. 187-196, 1998.

[11] E. D. Kaplan and C. J. Hegarty, Understanding the GPS: Principles and Applications, Artech House, 2006.

[12] C. Bettstetter, G. Resta and P. Santi, "The node distribution of the random waypoint mobility model for wireless ad-hoc networks," Mobile Computing, IEEE Transactions on, vol. 2, no. 3, pp. 257 269, 2003.

[13] S.-J. Lee, W. Su and M. Gerla, "On-Demand Multicast Routing Protocol in Multihop Wireless Mobile Networks," Mobile Networks and Applications, vol. 7, no. 6, pp. 441-453, 2002.

[14] C. E. Perkins and E. M. Royer, "Ad-hoc On Demand Distance Vector Routing," in IEEE WMCSA 99, New Orleans, 1999.

[15] Z. Wang, Y. Liang and L. Wang, "Multicast in Mobile ad-hoc Networks," Computer And Computing Technologies In Agriculture, vol. 258, no. 1, pp. 151-164, 2008.

Nermin Makhlouf was born in Damascus 1986. She was awarded the Degree of License in Electrical Engineering, department of Electronics and Telecommunications Engineering, Damascus University, Damascus, Syria in 2009.

She is a postgraduate student at Department of Telecommunications, Brno University of Technology, Brno, Czech Republic

Her research work has been concentrated on prediction of movement of wireless nodes in mobile ad-hoc networks MANETs. Recently she has also been concerned with routing protocols in mobile ad-hoc networks and how to overcome the interference and collision among nodes in such networks. 